\title{
EBSD Characterization of Materials: From Meteorites to Welds to MEMS
}

\author{
J.R. Michael ${ }^{1}$ and J. I. Goldstein ${ }^{2}$ \\ ${ }^{1}$ Sandia National Laboratories, PO Box 5800, Albuquerque, NM 87185-0886 \\ ${ }^{2}$ Mechanical and Industrial Engineering, University of Massachusetts, Amherst, MA 01003
}

Since the first high quality electron backscattered diffraction (EBSD) patterns were published 50 years ago, EBSD has become very useful for the characterization of polycrystalline materials.[1] The number of journal citations using some form of EBSD has increased from just a few in 1984 to close to 600 in 2003. EBSD has been used for both the identification of unknown phases and more commonly for automated orientation mapping where crystallography can be directly linked to microstructural features. Orientation mapping using EBSD is accomplished by obtaining EBSD patterns at an array of pixels on a sample. At each pixel the EBSD pattern is collected and automatically indexed and the crystallographic orientation calculated. A map of the crystallographic orientation (texture) of the sample can then be formed. Commercial EBSD systems now include the ability to map multi-phase materials and the identification of unknowns using large databases.

An example of the use of EBSD to solve an interesting crystallographic problem, for both scientific and commercial reasons, is the transformation of FCC to BCC in Fe-based alloy systems. An ideal opportunity to study this transformation is found in some meteorites as well as some inertial welds in austenitic alloys where both phases are present at room temperature. The use of orientation mapping allows the microstructure observed at room temperature to help understand the microstructure that existed at higher temperatures and the thermal history of the sample. Two examples of the FCC-BCC transformation are shown in Figure 1 and Figure 2. Figure 1a and b show orientation maps obtained from the meteorite for the FCC and BCC phases. The BCC phase shows many grains of different orientation while the FCC is all the same orientation. A probable explanation is that the parent body existed as FCC and then during cooling transformed via a martensitic reaction to $\mathrm{BCC}$ with retained regions of FCC as evidenced by the consistent orientation of the FCC throughout the sample. Figure $2 \mathrm{a}$ and $\mathrm{b}$ show similar maps for an inertial weld in an FCC alloy. Note that in this case the major phase is FCC with small amounts of BCC. The BCC exists as islands of BCC with similar orientations and shows that the inertial weld region transformed to $\mathrm{BCC}$ at high temperature with a grain size indicated by the areas of similar orientations.

EBSD has developed tremendously over the past 10 years in accuracy, speed and spatial resolution. The addition of EBSD systems to high current field emission SEM columns has allowed spatial resolutions that approach $20 \mathrm{~nm}$. Better sample preparation techniques, like FIB, allow higher resolution to be achieved as shown in Figure 3. Improvements in spatial resolution will also be possible with the availability of stable sample stages (reduced long term drift) combined with faster indexing algorithms. The continuing improvements and indicate a bright future for EBSD.[2]

\section{References}

[1] M. N. Alam et al., Proc. Roy. Soc. 221A (1954) 224.

[2] Sandia is a multiprogram laboratory operated by Sandia Corporation, a Lockheed Martin Company, for the United Stated Department of Energy (DOE) under contract DE-AC0494AL85000. 


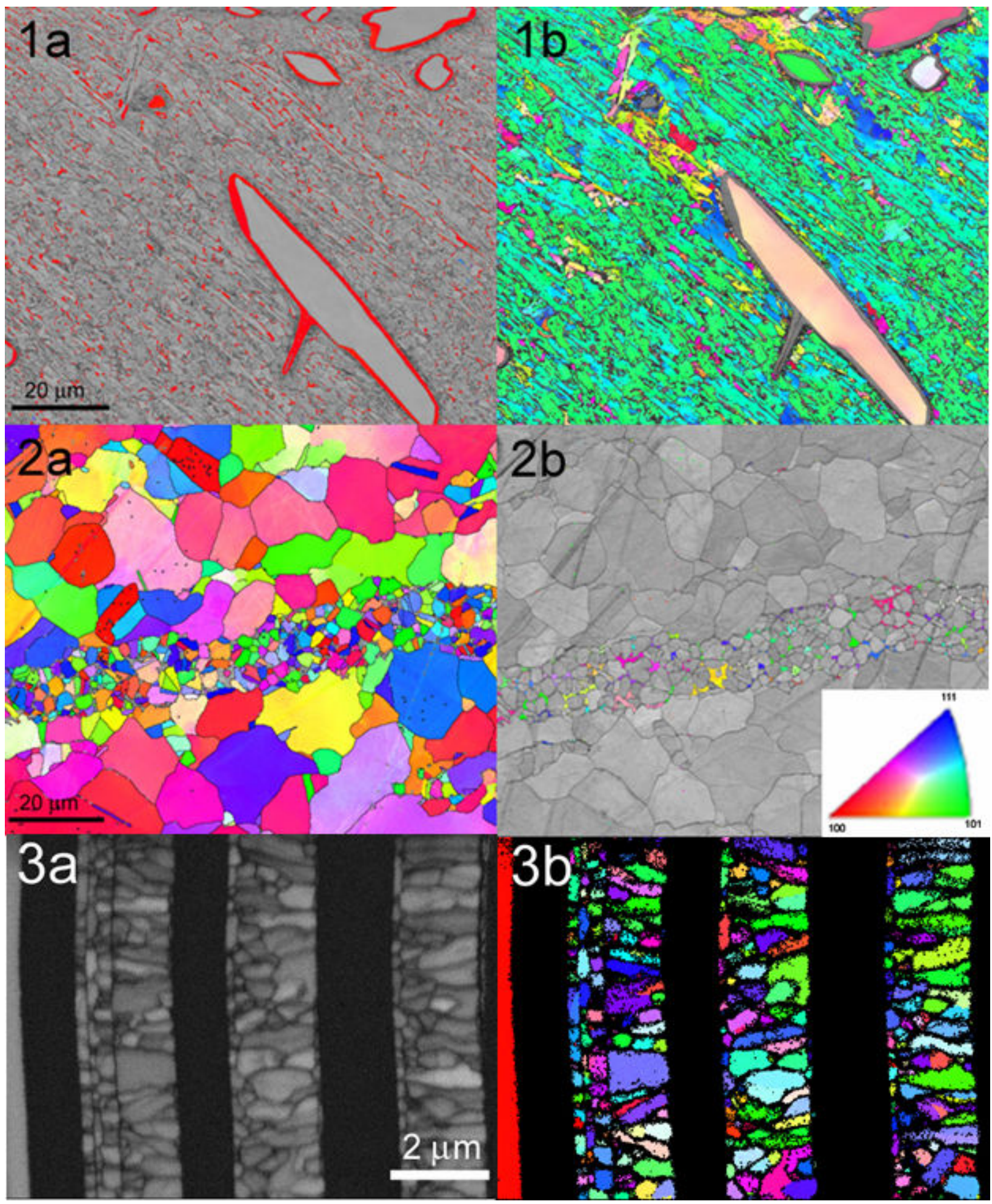

Figure 1. EBSD orientation maps of an Fe-Ni meteorite a)FCC phase, b) BCC phase.

Figure 2. Orientation maps of an inertial weld in an austenitic steel. a) FCC phase, b) BCC phase.

Figure 3. Orientation maps of polycrystalline Si layers prepared by FIB. A) Pattern contrast map, b) Orientation map of the polycrystalline Si obtained with a step size of $25 \mathrm{~nm}$ colored with respect to the growth direction. Stereographic triangle inset is used for orientation maps. 